\title{
Association Between Suggestive Symptom of Restless Legs Syndrome and COVID-19 Vaccination: A Pilot Study
}

\author{
Jin Myoung Seok ${ }^{1}$, Eun Jin $\mathrm{Na}^{1}$, Seul Gi Kim ${ }^{1}$, Jongkyu Park ${ }^{1}$, \\ Eunkyeong Park ${ }^{2}$, Pamela Song ${ }^{3}$, Kwang Ik Yang ${ }^{1}$ \\ ${ }^{1}$ Sleep Disorders Center, Department of Neurology, Soonchunhyang University Hospital Cheonan, \\ Soonchunhyang University College of Medicine, Cheonan, \\ ${ }^{2}$ Infection Control Team, Soonchunhyang University Hospital Cheonan, Cheonan, \\ ${ }^{3}$ Department of Neurology, Ilsan Paik Hospital, Inje University College of Medicine, Goyang, Korea
}

Received November 16, 2021 Revised December 14, 2021

Accepted December 20, 202

Address for correspondence Kwang Ik Yang, MD, PhD Sleep Disorders Center,

Department of Neurology,

Soonchunhyang University

Hospital Cheonan,

Soonchunhyang University

College of Medicine,

31 Soonchunhyang 6-gil,

Dongnam-gu,

Cheonan 31151, Korea

Tel: +82-41-570-2290

Fax: +82-41-592-3810

E-mail: neurofan@schmc.ac.kr
Objectives: Various sensory symptoms have been recognized after COVID-19 vaccination. Here, we aimed to explore the association between the suggestive symptom of restless legs syndrome (RLSss) and COVID-19 vaccination using an online survey. Methods: We prospectively studied participants who were working in our hospital after at least the first dose of the ChAdOx1 or BNT162b2 mRNA vaccine. The participants were invited via smartphone messages and voluntarily filled out an online questionnaire that included adverse events after vaccination. We considered the participants as having RLSss if they reported that they had three or more symptoms in the restless legs syndrome (RLS) diagnostic criteria. Results: A total of 628 participants ( 506 female; mean age, $37.7 \pm 12.4$ years) responded fully to our online survey. 588 participants (93.6\%) received the first dose of the ChAdOx1 vaccine (BNT162b2 mRNA vaccine for 40 participants). A total of 44 out of the 628 participants (7.0\%) reported that they had RLSss. Myalgia was more common in participants with RLSss than in those without RLSss (97.7\% vs. $67.3 \%, p<0.001)$. Multivariate testing showed that age (odds ratio, 1.037 per 1 year increase; 95\% CI, 1.004-1.071) and the presence of myalgia (odds ratio, 20.479; 95\% CI, 4.266-368.206) were associated with the presence of RLSss. Conclusions: This pilot study explored RLSss after COVID-19 vaccination and the results suggested that RLS might be one of the causes of adverse symptoms after COVID-19 vaccination. Further studies are required to confirm the relationship between RLS and COVID-19 vaccination.

J Sleep Med 2022;19(1):6-11

Keywords: COVID-19 vaccines; Restless legs syndrome; Adverse effects; Mass vaccination.

\section{INTRODUCTION}

Globally, national vaccination programs against severe acute respiratory coronavirus-2 (SARS-CoV-2) infection are in place. As the number of vaccinated people increases, safety concerns regarding coronavirus disease 2019 (COVID-19) vaccination have grown. Unexplained complications of COVID-19 vaccination have been revealed as a result of massive vaccination within a given period of time. The ChAdOx1 vaccine has been reported to be related to thrombotic immune thrombocytopenia, ${ }^{1}$ while the BNT162b2 mRNA and mRNA- 1273 vaccinations have recently been reported to be related to myocarditis, and pericarditis. ${ }^{2}$ The association between Guillain-Barre syn-

This is an Open Access article distributed under the terms of the Creative Commons Attribution Non-Commercial License (https://creativecommons.org/licenses/by-nc/4.0) which permits unrestricted non-commercial use, distribution, and reproduction in any medium, provided the original work is properly cited. drome (GBS) and COVID-19 vaccination has also been reported, ${ }^{3}$ but epidemiological studies have shown no conclusive results. ${ }^{4,5}$

In addition to these serious adverse events, common but modest symptoms after COVID-19 vaccination were also investigated; sensory symptoms including localized or generalized soreness, myalgia, itching, and tingling. ${ }^{6,7}$ However, clear explanations of these sensory symptoms are largely unavailable, and along with these symptoms, there is also an uncomfortable sensation in the lower extremities. These symptoms were reported to have remarkable similarity with symptoms of restless legs syndrome (RLS) by patients including one of the authors in this study. COVID-19 infection can affect the severity of RLS, but little is known about the relationship between RLS and COVID-19 vaccination. ${ }^{8}$

Here, we aimed to explore the association between the sug- 
gestive symptoms of RLS and COVID-19 vaccination using an online survey.

\section{METHODS}

\section{Participants and procedure}

The participants were healthcare employees working at our hospital (Soonchunhyang University Hospital Cheonan). All the participants were invited to participate in our survey via smartphone messages after the first or second dose of the ChAdOx1 or BNT162b2 mRNA vaccine, which were the only available vaccines at the time of study, and were administered according to the distribution protocol in Korea. The participants were informed about the goal and content of the study, and questionnaires were distributed to those who agreed to participate. The study was approved by the local ethics committee and the requirement for informed consent was waived (SCHCA 2021-09-019).

We conducted an online survey using Google Forms. The survey questionnaire included demographic features of the participants, self-reported current height and weight for calculating body mass index (BMI), type of COVID-19 vaccine, and adverse events after vaccination. The suggestive symptoms of RLS were investigated based on the four RLS diagnostic criteria: ${ }^{9} 1$ ) an urge to move the legs, usually accompanied or caused by uncomfortable and unpleasant sensations in the legs; 2) the urge to move or unpleasant sensations begin or worsen during periods of rest or inactivity such as lying down or sitting; 3) the urge to move or unpleasant sensations are partially or totally relieved by movement, such as walking or stretching, at least as long as the activity continues; and 4) the urge to move or unpleasant sensations are worse in the evening or night than during the day or only occur in the evening or night. The time interval between the onset of symptoms following vaccination and the severity of these symptoms was also evaluated using the visual analog scale (VAS). We considered participants with three or more symptoms from the RLS diagnostic criteria as having suggestive symptom of RLS (RLSss).

\section{Statistical analysis}

The demographic characteristics of the participants are presented with appropriate summary statistics. Continuous data are shown as means with standard deviations or medians with interquartile ranges (IQR). Categorical variables were presented as absolute and relative frequencies. We analyzed the differences between the groups (with or without RLSss) using a chi-square test or Fisher's exact test for categorical variables and Student's t-test or a Mann-Whitney U test for continuous variables. The Cochrane-Armitage trend test was used to evaluate the proportion of RLSss across the age range. Multivariate logistic regression analysis was performed to evaluate the independent contribution of the factors that influenced the presence of RLSss. Factors from univariate analyses were considered to represent explanatory variables and were evaluated using multivariate analysis, with the type of vaccine (either ChAdOx1 or BNT162b2 mRNA vaccine), age, sex, BMI, adverse symptoms, and the use of analgesics considered as predictors. Results are presented as odds ratios (ORs) with $95 \%$ confidence intervals (CI). Statistical significance was set at $p<0.05$. All statistical analyses were performed using R Programming software version 4.0.3 the ggplot2 package ( $\mathrm{R}$ Development Core Team, 2013; http://www.r-project.org).

\section{RESULTS}

\section{Demographic features}

A total of 628 participants completed all questions in the online survey, of which 506 were female (80.6\%). Their mean age was $37.7 \pm 12.4$ years, and their mean BMI was $22.3 \pm 3.3$ $\mathrm{kg} / \mathrm{m}^{2}$. The majority of the participants were vaccinated with the first dose of the ChAdOx1 vaccine (588, 93.6\%); only 40 participants were vaccinated with the BNT162b2 mRNA vaccine (first dose, $n=7$; second dose, $n=33$ ) (Fig. 1). Other demographic features of the groups are presented in Table 1.

\section{Characteristics of participants having 'suggestive symptom of RLS'}

A total of 44 of 628 participants (7.0\%) were classified as having RLSss with comparable symptoms of RLS. Among those with RLSss, $72.7 \%(\mathrm{n}=32)$ had no prior experience of symptoms, while $27.3 \%(n=12)$ had RLS symptoms previously. The proportion of participants with RLSss tended to increase with

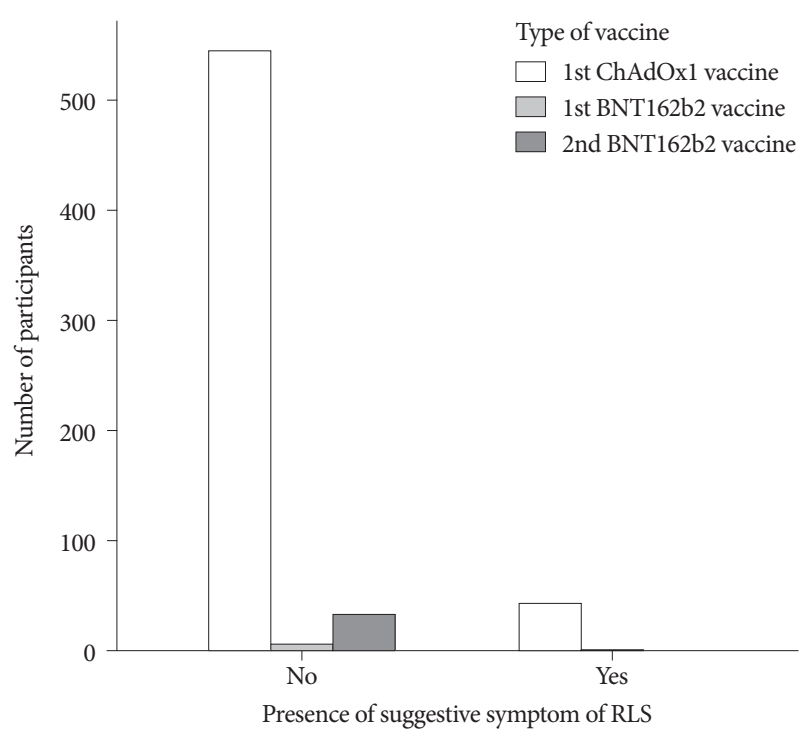

Figure 1. Types of vaccines administered to participants. RLS, restless legs syndrome. 
Table 1. Clinical characteristics of participants according to the presence of RLSss*

\begin{tabular}{|c|c|c|c|c|}
\hline & Total $(n=628)$ & With RLSss $(\mathrm{n}=44)$ & Without RLSss $(\mathrm{n}=584)$ & $p$ \\
\hline Age (yr) & $37.7 \pm 11.4$ & $39.9 \pm 12.0$ & $37.5 \pm 114$ & 0.418 \\
\hline Female & $506(80.6)$ & $38(86.4)$ & $468(80.1)$ & 0.181 \\
\hline BMI $\left(\mathrm{kg} / \mathrm{m}^{2}\right)$ & $22.3 \pm 3.3$ & $22.1 \pm 3.0$ & $22.3 \pm 3.3$ & 0.668 \\
\hline Vaccine type & & & & 0.349 \\
\hline ChAdOxl vaccine & $588(93.6)$ & $43(97.7)$ & $545(93.3)$ & \\
\hline BNT162b2 vaccine & $40(6.4)$ & $1(2.3)$ & $39(6.7)$ & \\
\hline \multicolumn{5}{|l|}{ Adverse symptoms } \\
\hline Dizziness & $194(30.9)$ & $12(27.3)$ & $182(31.2)$ & 0.712 \\
\hline Fever & $355(56.5)$ & $30(68.2)$ & $325(55.7)$ & 0.144 \\
\hline Headache & $341(54.3)$ & $26(59.1)$ & $315(53.9)$ & 0.614 \\
\hline Myalgia & $436(69.4)$ & $43(97.7)$ & $393(67.3)$ & $<0.001$ \\
\hline Injection site pain & $494(78.7)$ & $32(72.7)$ & $462(79.1)$ & 0.420 \\
\hline Use of analgesics & & & & 0.285 \\
\hline No analgesics & $96(15.3)$ & $3(6.8)$ & $93(15.9)$ & \\
\hline Before vaccination & $57(9.1)$ & $4(9.1)$ & $53(9.1)$ & \\
\hline After vaccination & $475(75.6)$ & $37(84.1)$ & $438(75.0)$ & \\
\hline Previous experience of RLSss & & $12(27.3)$ & & \\
\hline \multicolumn{5}{|l|}{ RLSss onset } \\
\hline The day at vaccination & & $20(45.5)$ & & \\
\hline First day after vaccination & & $14(31.8)$ & & \\
\hline Second day after vaccination & & $1(2.2)$ & & \\
\hline Other & & $9(20.5)$ & & \\
\hline \multicolumn{5}{|l|}{ Duration of RLSss } \\
\hline One day & & $11(25.0)$ & & \\
\hline Two days & & $16(36.4)$ & & \\
\hline Three days & & $11(25.0)$ & & \\
\hline Four or more days & & $6(13.6)$ & & \\
\hline Severity of RLSss & & $6.5 \pm 2.3$ & & \\
\hline
\end{tabular}

Data are presented as mean \pm standard deviation or $\mathrm{n}(\%) .{ }^{*}$ the participants were classified as having RLSss if they reported that there were 3 or more symptoms of restless legs syndrome diagnostic criteria. BMI, body mass index; RLSss, suggestive symptom of restless legs syndromes

age ( $p=0.235$, Cochrane-Armitage trend test) (Fig. 2). Symptoms appeared on the day of vaccination $(45.5 \%)$ or within 2 days of vaccination (79.5\%) in participants with RLSss. In the majority of participants (86.4\%), the symptoms improved within three days of symptom onset. The severity of symptoms, as assessed using the VAS, was $6.5 \pm 2.3$. The most commonly reported vaccination-related side effects were injection site pain, myalgia, fever, headache, and dizziness. Myalgia was more commonly reported in the participants with RLSss (97.7\% vs. $67.3 \%$, $p<0.001$ ) (Fig. 3). Most participants (84.7\%) chose to take analgesics to relieve various adverse symptoms, including RLSss, and the rate of analgesic use did not differ between the groups. Multivariate testing for independent factors that influenced the presence of RLSss showed that age (OR, 1.037 per 1 year increase; 95\% CI, 1.004-1.071) and the presence of myalgia (OR, 20.479; 95\% CI, 4.266-368.206) were independently associated with the presence of RLSss (Table 2).

\section{DISCUSSION}

In this study, we explored the relationship between the RLSss and COVID-19 vaccinations. We found that $7.0 \%$ of the participants reported RLSss after COVID-19 vaccination, which was related to older age and the presence of myalgia.

The diagnosis of RLS is based on clinical information, and without objective measurements, diagnostic uncertainty arises for obscure symptoms. ${ }^{10}$ Further diagnostic challenges can be investigated for questionnaire-based diagnosis of RLS; however, studies using self-reported questionnaires for the diagnosis of RLS showed comparable results of sensitivity and specificity compared to those of RLS diagnosis using expert interviews. ${ }^{11,12}$ Moreover, given the Diagnostic and Statistical Manual of Mental Disorders, 5th Edition (DSM-5) for RLS, short duration of RLSss in our participants may indicate different sensory complaints other than RLS symptoms; DSM-5 criteria include the 


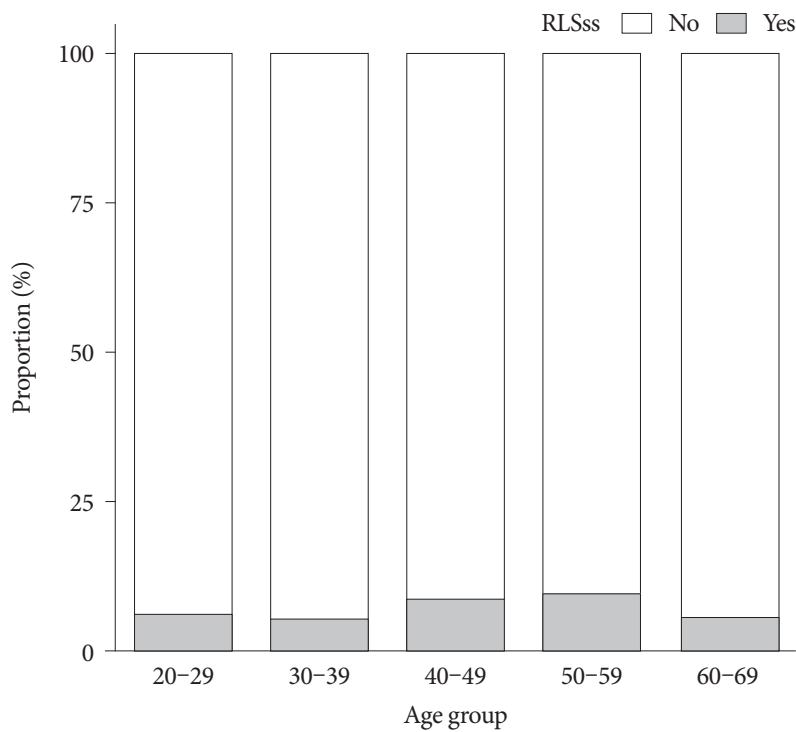

Figure 2. The proportions of 'RLSss' according to age groups. There was a tendency that the proportion of participants with RLSss increased according to the age ( $p=0.235$, by CochraneArmitage trend test). RLSss, suggestive symptom of restless legs syndrome.

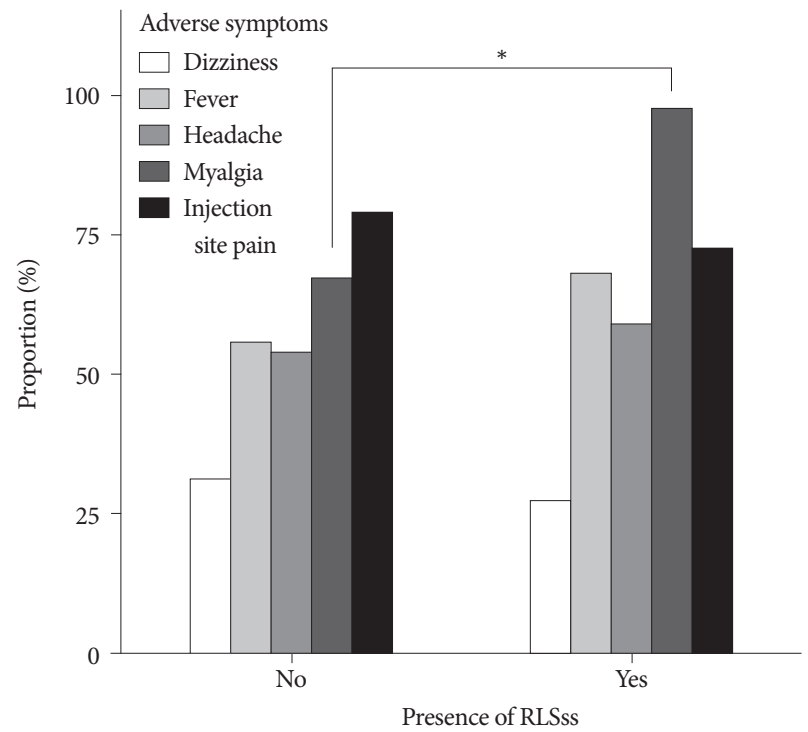

Figure 3. The proportions of adverse symptoms in participants according to the presence of RLSss. ${ }^{*} p<0.001$. RLSss, suggestive symptom of restless legs syndrome.

Table 2. Multivariate test for the presence of suggestive symptom of restless legs syndrome

\begin{tabular}{|c|c|c|c|}
\hline & Univariate OR $(95 \%$ CI $)$ & Multivariate OR $(95 \%$ CI $)$ & $p$ \\
\hline Age, per 1 year increase & $1.018(0.992-1.045)$ & $1.037(1.004-1.071)$ & 0.027 \\
\hline Female sex (vs. male) & $1.570(0.696-4.211)$ & & \\
\hline BMI, per $1 \mathrm{~kg} / \mathrm{m}^{2}$ increase & $0.978(0.884-1.075)$ & & \\
\hline \multicolumn{4}{|l|}{ Vaccine type } \\
\hline ChAdOxl vaccine & $3.077(0.643-55.255)$ & & \\
\hline BNT162b2 vaccine & Reference & & \\
\hline Use of analgesics before vaccination & $1.002(0.293-2.608)$ & & \\
\hline \multicolumn{4}{|l|}{ Adverse symptom } \\
\hline Dizziness & $0.828(0.402-1.604)$ & & \\
\hline Fever & $1.708(0.903-3.382)$ & & \\
\hline Headache & $1.234(0.666-2.333)$ & & \\
\hline Myalgia & $20.898(4.506-371.841)$ & $20.479(4.266-368.206)$ & 0.003 \\
\hline Injection site pain & $0.704(0.361-1.461)$ & & \\
\hline
\end{tabular}

BMI, body mass index; OR, odds ratio; CI, confidence interval

frequency and duration requirements. However, these variables are not included in the international RLS study group criteria because there could be a risk of underestimating the potential clinical significance of the intermittent subtype or recent-onset RLS. ${ }^{13}$ The online questionnaire in our study was based on RLS diagnostic criteria, and it could fairly detect true RLS symptoms.

Although the clinical trials of COVID-19 vaccines did not report major safety concerns, real-world data of COVID-19 vaccination showed various side-effects. ${ }^{14-16}$ Commonly reported adverse events include headache, fatigue, fever/chills, diarrhea/nausea, myalgia/arthralgia, and various sensory symp- toms. ${ }^{17,18}$ Some authors have proposed that these sensory symptoms are caused by an atypical type of GBS involving mainly sensory nerves, ${ }^{19}$ and others reported that small fiber neuropathy could occur after COVID-19 vaccination. ${ }^{20}$ While the origins of these sensory symptoms are mostly undiscovered, some sensory symptoms are similar to those of RLS. Furthermore, to the best of our knowledge, RLS-like symptoms associated with COVID-19 vaccination have not been previously studied.

In our study, most participants with RLSss $(n=32,72.7 \%)$ had never had such symptoms before, and symptoms resolved within 3 days ( $n=38,86.4 \%)$. This suggests that unpleasant sensory symptoms following COVID-19 vaccination may suggest 
a new onset of RLS. The transient characteristics of our participants' symptoms may suggest that COVID-19 vaccination may have provoked or lowered the threshold for RLS symptoms in participants with subclinical RLS or previous RLS, and most of the participants with previous symptoms of RLS ( $n=12$, $27.3 \%$ ) also reported that their symptoms were relieved within 3 days $(n=9,75.0 \%)$.

The provocation of RLS following COVID-19 vaccination can be attributed to the vaccination itself as well as to weariness, fatigue, or myalgia. Patients diagnosed with RLS commonly report fluctuation of symptoms depending on the exposure to risk factors, such as iron deficiency, medications, or infection. ${ }^{21,22}$ It is not clear which was the major contributor to the trigger of RLS symptoms. However, the significant coexistence of myalgia with RLSss, along with short RLSss duration, suggests that acute immune reaction and local inflammation may have played a role. Further studies are required to confirm the relationship between RLS and COVID-19 vaccination.

The clarification of the origins of adverse events after vaccination is important as the experience of unexpected symptoms after vaccination could lead to negative attitudes and reluctant behaviors toward vaccines. The information on safety and reactogenicity profiles of the vaccines could help promote vaccinations and reduce vaccination-related anxiety. ${ }^{23}$ With this point of view, the safety and reactogenicity profiles are actively researched in many clinical trials of vaccines, and our study can be a part of this effort to elucidate the origins of adverse symptoms after vaccination. We conducted this study via an online survey using smartphones to collect data from the participants, which could be a useful tool in this COVID-19 era. ${ }^{24}$ However, further studies of the correlation between smartphone survey and face-to-face interviews for evaluating RLS are required.

Our study had several limitations. First, we used voluntarily self-reported data via an online survey using a smartphone, which can have potential risks of errors in omission and response bias; some participants might be more inclined to report symptoms than others. Second, because this was a crosssectional study involving healthcare staff in a single tertiary facility, the demographic profiles of the participants were not controlled, limiting the potential for the generalization of our findings. Finally, there was no control over the type of vaccine used or the timing of the online survey administered after vaccination. However, this was a pilot study, which represents the first effort to clarify the relationship between RLS and COVID-19 vaccination. Further large-scale studies with controlled populations are required.

In conclusion, RLSss can occur after COVID-19 vaccination, and we suggest that RLS might be one of the causes of various adverse symptoms after COVID-19 vaccination. Further studies are required to confirm the relationship between RLS and
COVID-19 vaccination.

\section{Conflicts of Interest}

The authors have no potential conflicts of interest to disclose.

\section{ORCID iDs}

$\begin{array}{ll}\text { Jin Myoung Seok } & \text { https://orcid.org/0000-0002-1484-2968 } \\ \text { Eun Jin Na } & \text { https://orcid.org/0000-0002-0464-9902 } \\ \text { Seul Gi Kim } & \text { https://orcid.org/0000-0002-9065-0781 } \\ \text { Jongkyu Park } & \text { https://orcid.org/0000-0002-9261-790X } \\ \text { Eunkyeong Park } & \text { https://orcid.org/0000-0001-8044-8275 } \\ \text { Pamela Song } & \text { https://orcid.org/0000-0002-9750-5683 } \\ \text { Kwang Ik Yang } & \text { https://orcid.org/0000-0001-6343-6520 }\end{array}$

\section{Author Contributions}

Conceptualization: Jin Myoung Seok, Kwang Ik Yang. Data curation: Eun Jin Na, Seul Gi Kim, Jongkyu Park, Eunkyeong Park, Kwang Ik Yang. Formal analysis: Jin Myoung Seok, Kwang Ik Yang. Investigation: Jin Myoung Seok, Kwang Ik Yang. Methodology: Jin Myoung Seok, Kwang Ik Yang. Project administration: Jin Myoung Seok, Eunkyeong Park, Kwang Ik Yang. Supervision: Kwang Ik Yang. Visualization: Jin Myoung Seok. Writing-original draft: Jin Myoung Seok, Kwang Ik Yang. Writing-review \& editing: Jin Myoung Seok, Pamela Song, Kwang Ik Yang.

\section{Funding Statement}

None

\section{Acknowledgments}

We are very grateful to our colleagues in Soonchunhyang University Hospital Cheonan for their contribution to this study.

\section{REFERENCES}

1. Cines DB, Bussel JB. SARS-CoV-2 vaccine-induced immune thrombotic thrombocytopenia. N Engl J Med 2021;384:2254-2256. https:// doi.org/10.1056/NEJMe2106315.

2. Larson KF, Ammirati E, Adler ED, et al. Myocarditis after BNT162b2 and mRNA-1273 vaccination. Circulation 2021;144:506-508. https:// doi.org/10.1161/CIRCULATIONAHA.121.055913.

3. Maramattom BV, Krishnan P, Paul R, et al. Guillain-Barré syndrome following ChAdOx1-S/nCoV-19 vaccine. Ann Neurol 2021;90:312-314. https://doi.org/10.1002/ana.26143.

4. Lunn MP, Cornblath DR, Jacobs BC, et al. COVID-19 vaccine and Guillain-Barré syndrome: let's not leap to associations. Brain 2021;144: 357-360. https://doi.org/10.1093/brain/awaa444.

5. Keddie S, Pakpoor J, Mousele C, et al. Epidemiological and cohort study finds no association between COVID-19 and Guillain-Barré syndrome. Brain 2021;144:682-693. https://doi.org/10.1093/brain/awaa433.

6. Kadali RAK, Janagama R, Peruru S, Malayala SV. Side effects of BNT162b2 mRNA COVID-19 vaccine: a randomized, cross-sectional study with detailed self-reported symptoms from healthcare workers. Int J Infect Dis 2021;106:376-381. https://doi.org/10.1016/j.ijid.2021.04.047.

7. Park C, Sakong J, Jo S, Kim M, Baek K. Adverse effects on work and daily life interference among healthcare workers after the first and second ChAdOx1 and BNT162b2 COVID-19 vaccine doses. Vaccines (Basel) 2021;9:926. https://doi.org/10.3390/vaccines9080926.

8. Franco B, Morais MA, Holanda ASS, Manconi M, de Mello MT, Esteves AM. Impact of Covid-19 on the restless legs syndrome. Sleep Sci 2020;13:186-190. https://doi.org/10.5935/1984-0063.20200031.

9. Allen RP, Picchietti D, Hening WA, Trenkwalder C, Walters AS, Montplaisi J. Restless legs syndrome: diagnostic criteria, special considerations, and epidemiology. A report from the restless legs syndrome diagnosis and epidemiology workshop at the National Institutes of Health. Sleep 
Med 2003;4:101-119. https://doi.org/10.1016/s1389-9457(03)00010-8.

10. Hening WA, Allen RP, Washburn M, Lesage SR, Earley CJ. The four diagnostic criteria for restless legs syndrome are unable to exclude confounding conditions (“mimics"). Sleep Med 2009;10:976-981. https:// doi.org/10.1016/j.sleep.2008.09.015.

11. Allen RP, Burchell BJ, MacDonald B, Hening WA, Earley CJ. Validation of the self-completed Cambridge-Hopkins questionnaire (CHRLSq) for ascertainment of restless legs syndrome (RLS) in a population survey. Sleep Med 2009;10:1097-1100. https://doi.org/10.1016/ j.sleep.2008.10.007.

12. Popat RA, Van Den Eeden SK, Tanner CM, et al. Reliability and validity of two self-administered questionnaires for screening restless legs syndrome in population-based studies. Sleep Med 2010;11:154-160. https://doi.org/10.1016/j.sleep.2009.01.012.

13. Allen RP, Picchietti DL, Garcia-Borreguero D, et al. Restless legs syndrome/Willis-Ekbom disease diagnostic criteria: updated International Restless Legs Syndrome Study Group (IRLSSG) consensus criteria-history, rationale, description, and significance. Sleep Med 2014;15: 860-873. https://doi.org/10.1016/j.sleep.2014.03.025.

14. Falsey AR, Sobieszczyk ME, Hirsch I, et al. Phase 3 safety and efficacy of AZD1222 (ChAdOx1 nCoV-19) Covid-19 vaccine. N Engl J Med 2021;385:2348-2360. https://doi.org/10.1056/NEJMoa2105290.

15. Polack FP, Thomas SJ, Kitchin N, et al. Safety and efficacy of the BNT162b2 mRNA Covid-19 vaccine. N Engl J Med 2020;383:26032615. https://doi.org/10.1056/NEJMoa2034577.

16. Baden LR, El Sahly HM, Essink B, et al. Efficacy and safety of the mRNA-1273 SARS-CoV-2 vaccine. N Engl J Med 2021;384:403-416.
https://doi.org/10.1056/NEJMoa2035389.

17. Mathioudakis AG, Ghrew M, Ustianowski A, et al. Self-reported realworld safety and reactogenicity of covid-19 vaccines: a vaccine recipient survey. Life (Basel) 2021;11:249. https://doi.org/10.3390/life11030249.

18. Menni C, Klaser K, May A, et al. Vaccine side-effects and SARS-CoV-2 infection after vaccination in users of the COVID symptom study app in the UK: a prospective observational study. Lancet Infect Dis 2021; 21:939-949. https://doi.org/10.1016/S1473-3099(21)00224-3.

19. Min YG, Ju W, Ha YE, et al. Sensory Guillain-Barre syndrome following the ChAdOx1 nCov-19 vaccine: report of two cases and review of literature. J Neuroimmunol 2021;359:577691. https://doi.org/10.1016/ j.jneuroim.2021.577691.

20. Waheed W, Carey ME, Tandan SR, Tandan R. Post COVID-19 vaccine small fiber neuropathy. Muscle Nerve 2021;64:E1-E2. https://doi. org/10.1002/mus.27251.

21. Weinstock LB, Walters AS, Paueksakon P. Restless legs syndrome-theoretical roles of inflammatory and immune mechanisms. Sleep Med Rev 2012;16:341-354. https://doi.org/10.1016/j.smrv.2011.09.003.

22. Guo S, Huang J, Jiang H, et al. Restless legs syndrome: from pathophysiology to clinical diagnosis and management. Front Aging Neurosci 2017;9:171. https://doi.org/10.3389/fnagi.2017.00171.

23. Hervé C, Laupèze B, Del Giudice G, Didierlaurent AM, Tavares Da Silva $\mathrm{F}$. The how's and what's of vaccine reactogenicity. NPJ Vaccines 2019;4:39. https://doi.org/10.1038/s41541-019-0132-6.

24. Budd J, Miller BS, Manning EM, et al. Digital technologies in the public-health response to COVID-19. Nat Med 2020;26:1183-1192. https://doi.org/10.1038/s41591-020-1011-4. 